-Review

\title{
Influence of deep brain stimulation of the subthalamic nucleus on cognitive function in patients with Parkinson's disease
}

\author{
Bin $\mathrm{Wu}^{1}$, Lu Han ${ }^{1}$, Bo-Min Sun ${ }^{2}$, Xiao-Wu Hu ${ }^{3}$, Xiao-Ping Wang ${ }^{1}$ \\ ${ }^{1}$ Department of Neurology, Shanghai First People's Hospital, School of Medicine, Shanghai Jiao Tong University, Shanghai \\ 200080, China \\ ${ }^{2}$ Department of Functional Neurosurgery, Ruijin Hospital Affiliated to the School of Medicine, Shanghai Jiao Tong University, \\ Shanghai 200025, China \\ ${ }^{3}$ Department of Neurosurgery, Changhai Hospital, Second Military University, Shanghai 200433, China \\ Corresponding author: Xiao-Ping Wang. E-mail: wangxp@ustc.edu
}

(C) Shanghai Institutes for Biological Sciences, CAS and Springer-Verlag Berlin Heidelberg 2014

Deep brain stimulation (DBS) is an effective technique for treating Parkinson's disease (PD) in the middle and advanced stages. The subthalamic nucleus (STN) is the most common target for clinical treatment using DBS. While STN-DBS can significantly improve motor symptoms in PD patients, adverse cognitive effects have also been reported. The specific effects of STN-DBS on cognitive function and the related mechanisms remain unclear. Thus, it is imperative to identify the influence of STN-DBS on cognition and investigate the potential mechanisms to provide a clearer view of the various cognitive sequelae in PD patients. For this review, a literature search was performed using the following inclusion criteria: (1) at least 10 patients followed for a mean of at least 6 months after surgery since the year 2006; (2) pre- and postoperative cognitive data using at least one standardized neuropsychological scale; and (3) adequate reporting of study results using means and standard deviations. Of $\sim 170$ clinical studies identified, 25 cohort studies (including 15 self-controlled studies, nine intergroup controlled studies, and one multi-center, randomized control experiment) and one metaanalysis were eligible for inclusion. The results suggest that the precise mechanism of the changes in cognitive function after STN-DBS remains obscure, but STN-DBS certainly has effects on cognition. In particular, a progressive decrease in verbal fluency after STN-DBS is consistently reported and although executive function is unchanged in the intermediate stage postoperatively, it tends to decline in the early and later stages. However, these changes do not affect the improvements in quality of life. STN-DBS seems to be safe with respect to cognitive effects in carefully-selected patients during a follow-up period from 6 months to 9 years.

Keywords: Parkinson's disease; subthalamic nucleus; deep brain stimulation; cognitive function; mechanism

\section{Introduction}

Parkinson's disease (PD) is the second most common neurodegenerative disorder after Alzheimer's disease. It is characterized by classical motor features (resting tremor, rigidity, bradykinesia, and postural instability) among middle-aged and elderly individuals. Medication to improve the movement-related symptoms is the preferred method of treatment. Deep brain stimulation (DBS) is a novel and effective treatment in the middle and advanced stages of PD. DBS involves implanting microelectrodes in specific nuclei and applying pulse generator-induced electrical stimulation. The subthalamic nucleus (STN), medial globus pallidus, and nucleus ventralis intermedius thalami are common targets for DBS treatment. Among these targets, STN-DBS typically results in significantly improved tremor 
and rigidity, ameliorates bradykinesia, and has positive effects on postural instability. Moreover, STN-DBS has been found to reduce the necessary dose of postoperative dopaminergic drugs ${ }^{[1]}$, so the STN has become the most common target ${ }^{[1,2]}$. While STN-DBS has been found to improve movement symptoms, complications in cognitive function have also been reported ${ }^{[3]}$. A meta-analysis reported that $\sim 41 \%$ of $P D$ patients present with cognitive disorders after $\mathrm{DBS}^{[4]}$. In recent years, increasing numbers of studies have focused on the influence of STN-DBS on cognitive function. However, its specific impact on cognitive function in PD patients and its precise mechanisms remain poorly understood. Thus, it is imperative to identify the influence of STN-DBS on cognition and investigate its potential mechanisms to provide a clearer understanding of the various cognitive sequelae in PD patients.

Here, we selected and reviewed research on the influence of STN-DBS on cognitive function in PD patients by setting the following inclusion criteria: (1) at least 10 patients followed for a mean of at least 6 months after surgery during the 6 years from June 2006 to June 2012; (2) pre- and post-operative cognitive data using at least one standardized neuropsychological scale; and (3) adequate reporting of study results using means and standard deviations. We searched the PubMed and Cochrane Library databases for articles written in English, and the Chinese Knowledge Resource Integrated (CNKI) and Wanfang databases for articles written in Chinese, using the key search terms "deep brain stimulation, Parkinson's disease, cognition OR cognitive" in English, and "DBS, PD" in Chinese. About 170 papers were identified, of which 25 cohort studies (15 self-controlled studies, nine intergroupcontrolled studies, and one multi-center, randomized control experiment) and one meta-analysis were eligible for inclusion.

The reason we only focused on papers after 2006 was mainly that an authoritative meta-analysis had been published in $2006^{[1]}$. But ours differs considerably from that report. Primarily, most of the studies in that meta-analysis did not have control groups and were not randomized clinical trials, which limited the interpretation of whether cognitive decline was directly associated with or caused by DBS. Here, we included more controlled studies and even a multi-center, randomized control experiment, so the data are comparatively more convincing. Moreover, compared with the previous studies, those of the last 6 years have a more comprehensive perspective on the cognitive domain. In addition, we included an exploration of possible mechanisms underlying the changes of cognition after DBS, which was absent from the previous report.

By comparing these 26 studies, we summarize the influence of STN-DBS on cognitive function in PD patients and investigate the possible underlying mechanisms from analyses of speech, execution, intelligence, memory, attention, spatial visual function, psychomotor speed, and other domains (Table 1).

\section{Influence of STN-DBS on Cognitive Function in Patients with PD}

\section{Verbal Fluency}

Verbal fluency has been extensively used as a standardized neuropsychological scale, and includes phonemic and semantic fluency (also referred to as 'letter fluency' and 'category fluency'). A large number of follow-up studies have investigated the influence of DBS on verbal fluency in PD patients after surgery. In the follow-up of self-controls before and after treatment, verbal fluency was reduced in all studies except one in which it remained unchanged ${ }^{[16]}$. In particular, phonemic fluency was significantly reduced ${ }^{[9,12]}$, while semantic fluency remained unchanged. Gaspari et al. ${ }^{[6]}$ quantitatively and qualitatively analyzed vocabulary number, vocabulary switching, and vocabulary cluster size, and found that the total number of words and switches decreased, while the average cluster size was unchanged. York et al. ${ }^{[21]}$ conducted a controlled study with 6-month follow-up involving 23 PD patients treated with STN-DBS, and 28 PD patients receiving drug treatment. The results revealed that semantic fluency declined after surgery. However, the control group also exhibited a decline and there was no significant difference between the groups, consistent with the results of another previous study ${ }^{[27]}$. Three follow-up studies containing non-surgical control groups also revealed reduced verbal fluency ${ }^{[20,23,26]}$. Witt et al. ${ }^{[29]}$ conducted a multicenter randomized control study to investigate the cognitive changes in 60 PD patients undergoing STN-DBS in 2008, and reported that both phonetic and semantic fluency were reduced. A meta- 
Table 1. Summary of effects of STN-DBS on cognitive function in PD patients (since 2006)

\begin{tabular}{|c|c|c|c|c|c|c|c|c|}
\hline \multirow[t]{2}{*}{ Authors (year) } & \multirow{2}{*}{$\begin{array}{l}\text { Case } \\
\text { number }\end{array}$} & \multirow{2}{*}{$\begin{array}{c}\text { Surgery } \\
\text { age } \\
\text { (year) }\end{array}$} & \multirow{2}{*}{$\begin{array}{c}\text { Course of } \\
\text { disease } \\
\text { (year) }\end{array}$} & \multirow{2}{*}{$\begin{array}{c}\text { Education } \\
\text { level } \\
\text { (year) }\end{array}$} & \multirow{2}{*}{$\begin{array}{l}\text { Follow-up } \\
\text { duration } \\
\text { (month) }\end{array}$} & \multicolumn{3}{|c|}{ Cognitive function } \\
\hline & & & & & & Reduced & Elevated & Unchanged \\
\hline \multicolumn{9}{|l|}{ Cohort study (self-control) } \\
\hline Castelli et al. $(2006)^{[5]}$ & 72 & 60.5 & 15.1 & 8.7 & 15 & VF & - & $\mathrm{E}, \mathrm{M}$ \\
\hline Gaspari et al. $(2006)^{[6]}$ & 20 & 59.8 & 15.8 & 11.4 & 15 & VF & - & VCS \\
\hline Erola et al. $(2006)^{[7]}$ & 29 & 60.0 & 13.0 & $N R$ & 12 & VF & - & 1 \\
\hline Aybek et al. $(2007)^{[8]}$ & 57 & 63.8 & 15.5 & NR & 36 & $V F, A, E, M, C$ & - & SVF \\
\hline Castelli et al. $(2007)^{[9]}$ & 19 & 62.1 & 14.7 & $N R$ & 17 & VF-PF ${ }^{a}$ & - & VF-SF ${ }^{a}, M, E$ \\
\hline Ory-Magne et al. $(2007)^{[10]}$ & 45 & 60.1 & 13.5 & NR & 24 & VF & - & $E$ \\
\hline Rothlind et al. (2007) ${ }^{[11]}$ & 19 & 61.4 & 12.9 & 15.2 & 15 & VF, M & - & $E$ \\
\hline Contarino et al. $(2007)^{[12]}$ & 11 & 57.5 & 15.5 & 11.0 & 60 & VF-PF, E & $\mathrm{I}^{\mathrm{b}}$ & M \\
\hline Klempirova et al. $(2007)^{[13]}$ & 19 & 57.0 & 15.0 & NR & 12 & VF, M, E & - & PSM \\
\hline Heo et al. $(2008)^{[14]}$ & 46 & 57.9 & 11.6 & 8.0 & 12 & $V F, M, E(S t r o o p)^{c}$ & - & $\mathrm{A}, \mathrm{E}(\mathrm{WCST})^{\mathrm{c}}$ \\
\hline Fasano et al. $(2010)^{[15]}$ & 20 & 56.7 & 13.7 & NR & 96 & VF, E, M & - & - \\
\hline Kishore et al. $(2010)^{[16]}$ & 45 & 55.4 & 11.1 & NR & 60 & - & - & VF, M, E \\
\hline Merola et al. $(2011)^{[17]}$ & 19 & 61.5 & 22.8 & NR & 95 & VF, E, M & - & - \\
\hline Zibetti et al. $(2011)^{[18]}$ & 47 & 61.4 & 16.4 & NR & $\sim 108$ & $V F, E, M, A^{e}$ & - & - \\
\hline Yamanaka et al. $(2012)^{[19]}$ & 30 & 61.0 & 11.5 & 12.5 & 12 & - & - & $(V F, E)^{d}$ \\
\hline \multicolumn{9}{|l|}{ Cohort study (intergroup control) } \\
\hline Smeding et al. $(2006)^{[20]}$ & 99 & 57.9 & 13.7 & 11.0 & 6 & VF, M, A, E & - & PMS, SVF \\
\hline York et al. $(2008)^{[21]}$ & 23 & 59.5 & 12.0 & 14.4 & 6 & M & - & (VF-SF, A, SS) \\
\hline Zahodne et al. $(2009)^{[22]}$ & 22 & NR & NR & NR & 12 & VF & - & M \\
\hline Zangaglia et al. $(2009)^{[23]}$ & 32 & 58.8 & 11.8 & 7.3 & 36 & $V F, E^{g}$ & - & M \\
\hline Castelli et al. $(2010)^{[24]}$ & 27 & 60.6 & 15.3 & 8.0 & 12 & VF-PF & - & $\mathrm{E}, \mathrm{M}$ \\
\hline Mikos et al. $(2010)^{[25]}$ & 24 & 61.7 & 12.0 & 14.1 & 16 & M, PSM, E & SVF & - \\
\hline Smeding et al. $(2011)^{[26]}$ & 105 & 58.4 & 13.0 & 11.1 & 12 & VF, M, A, E & - & - \\
\hline Williams et al. $(2011)^{[27]}$ & 19 & 62.1 & 13.6 & 10.1 & 24 & $M$ & - & $(V F, E, S V F)^{h}$ \\
\hline Zangaglia et al. $(2012)^{[28]}$ & 30 & 58.8 & 11.8 & 7.3 & 96 & PF & - & $\mathrm{M}, \mathrm{E}$ \\
\hline \multicolumn{9}{|l|}{ Randomized control } \\
\hline Witt et al. $(2008)^{[29]}$ & 60 & 60.2 & 13.8 & NR & 6 & VF, E & - & $A, M$ \\
\hline \multicolumn{9}{|l|}{ Meta analysis } \\
\hline Parsons et al. $(2006)^{[4]}$ & 612 & - & - & - & - & VF, E, M & - & - \\
\hline
\end{tabular}

NR, Not reported; A, attention; E, execution; SS, set-shifting; I, intelligence; M, memory; PMS, psychomotor speed; VF, verbal fluency; PF, phonetic

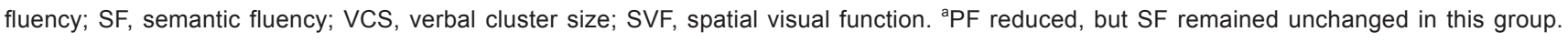
${ }^{b}$ intelligence estimated using the mini-mental state examination; scores were significantly elevated at 1-year follow-up compared with baseline, but returned to baseline at 5 years. ${ }^{~}$ executive function: Stroop test performance declined, but WCST performance remained unchanged. ${ }^{d} V F$ and $E$ reduced at 1 month postoperatively, but returned to normal at 12 months. ${ }^{e}$ attention transfer evaluated using trail-making test B: scores significantly elevated at 1-year follow-up compared with baseline, reduced to baseline at 5 years, and continued to decrease up to 9 years later. ${ }^{\mathrm{f}} \mathrm{SF}$, A, and SS reduced after treatment, but the decrease was not significantly different from the control group. 9frontal lobe E reduced at 1 month of follow-up, and returned to normal at 12 months. ${ }^{\mathrm{h}} \mathrm{E}, \mathrm{SVF}$, and VF reduced after operation, as was the control group; there was no significant difference between groups. 
analysis of 28-item cohort studies involving 612 PD patients in $2006{ }^{[4]}$ reported that phonetic and semantic fluency were reduced. This finding was not related to age, course of disease, stimulation parameters, or postsurgical dopamine dose.

In addition, a long-term follow-up study of verbal fluency reported that $30-50 \%$ of patients exhibited significantly reduced verbal fluency at 3 years after surgery ${ }^{[30]}$. However, verbal fluency was found to be marginally reduced at 1 year, and significantly reduced at 5 years after bilateral STN-DBS in 11 PD patients ${ }^{[12]}$, consistent with the Fasano study ${ }^{[15]}$ in which verbal fluency was significantly reduced at 8 years compared with 5 years in 20 PD patients. These results reflect a progressive reduction of verbal fluency after surgery.

Overall, from self-controlled and intergroup-controlled studies, a multi-center, randomized control study, and further meta-analysis, verbal fluency is consistently reported to progressively decrease after STN-DBS. Some studies also reported a separation of phonetic and semantic fluency.

\section{Executive Function}

Executive function is a cognitive process that controls and monitors individual consciousness and behavior, such as planning, working memory, attention, problem-solving, verbal reasoning, inhibition, mental flexibility, multi-tasking, and the initiation and monitoring of actions. Opinions as to the specific effects of STN-DBS on executive function are largely divided.

First, most reported studies suggest that STN-DBS decreases executive function. A 6-month follow-up study of 99 PD patients receiving STN-DBS revealed reductions in interference control and set-shifting in PD patients compared with a control group, as tested by the Stroop test and trail-making test $\mathrm{B}^{[20]}$. Aybek et al. ${ }^{[8]}$ followed $57 \mathrm{PD}$ patients for 3 years, reporting that STN-DBS decreased executive function, specifically inhibition, initiation, and reasoning. A randomized controlled study ${ }^{[29]}$ and a metaanalysis ${ }^{[4]}$ also supported this result.

Second, several studies have reported that executive function does not change after STN-DBS. For example, Raven's progressive matrices, trail-making test $B$, and the Wisconsin card-sorting test were used to assess executive function in 19 patients during a 17-month follow- up, revealing no change ${ }^{[9]}$. Another study reported that executive function tended to decrease after STN-DBS, but remained similar to the control group ${ }^{[27]}$.

Third, some opinions of the changes of executive function after STN-DBS are mixed. For example, one study reported that Raven's progressive matrices test revealed that abstract reasoning was significantly reduced after STN-DBS, while performance in the Wisconsin card-sorting test was unchanged ${ }^{[12]}$, consistent with Heo's study of 46 $\mathrm{PD}$ patients followed up for 1 year ${ }^{[14]}$. Zangaglia et al. ${ }^{[23]}$ conducted a 3-year follow-up of 32 PD patients with STNDBS and 33 treated with drugs, and found that logic-related executive function was reduced one month postoperatively, returned to the normal range at 6 months, and was comparable to that prior to surgery at one year. These findings indicate that the reduced frontal lobe executive function is transient. The authors then followed the same patients for up to 8 years and found that only phonetic fluency was significantly reduced in the STN-DBS group compared with a non-surgery group, while no difference was found in executive function or memory between groups $^{[28]}$.

Therefore, the influence of STN-DBS on executive function seems elusive. The reasons are as follows: (1) different studies used different neuropsychological scales with variable sensitivity ${ }^{[10]}$. For example, in testing executive function, the Wisconsin card-sorting test focuses on abstract abilities, concept formation, and the ability to shift, while the Stroop color-word test focuses on interference control; and (2) different experimental designs may result in different experimental effects. For example, in a cohort study with pre- and post-operative self-control, it is difficult to identify whether positive results are from surgery or disease progression; in studies with surgical and nonsurgical groups, unless the grouping is random, sampling errors and bias factors cannot be eliminated. However, some conclusions can be drawn. As shown in Table 1, the follow-up duration of studies reporting decreased executive function are typically relatively short $(<6 \text { months })^{[19,23]}$ or relatively long ( $>5$ years), while those reporting unchanged executive function usually have an intermediate duration of follow-up (1-2 years). The early, transient reduction of executive function following STN-DBS may implicate microinjury in surgery, in accord with the finding that executive 
function recovers and remains stable after injury adaptation and repair. The progression of PD over time may be another factor associated with a further decline of executive function.

\section{Intelligence and Memory}

The effects of STN-DBS on intelligence and memory remain unclear. A meta-analysis ${ }^{[4]}$ and several clinical studies $^{[14,26]}$ reported significantly reduced verbal memory, particularly delayed recall, after surgery. Fasano et al. ${ }^{[15]}$ reported that episodic memory was significantly reduced after surgery in an 8-year follow-up using the Rey auditoryverbal learning test. However, Contarino et al. ${ }^{[12]}$ reported that episodic and short-term memory were decreased, but not to a statistically significant level. A study using the Wechsler memory scale-III reported that declarative memory, including immediate memory, delayed memory, and recognition, was significantly reduced in patients subjected to STN-DBS ${ }^{[13]}$. In contrast, another study showed that immediate and episodic memory were not substantially affected by STN-DBS in a randomized control experiment ${ }^{[29]}$. This divergence may result from different scale structures and sensitivities as well as varied follow-up durations.

The Mini-Mental State Examination (MMSE) is commonly used to evaluate "general intelligence", although this is a vague concept and is better evaluated by its components. It is worth mentioning that one study administered the MMSE to patients undergoing STNDBS, reporting that intelligence was significantly elevated at 1 year after surgery compared with baseline, but was reduced to baseline levels at up to 5 years $^{[12]}$. We suggest that this difference was due to a practice effect in the DBS group.

A 3-year follow-up of 57 PD patients undergoing STNDBS found that $24.5 \%$ developed dementia, an incidence similar to that of medically-treated patients ${ }^{[8]}$. Another study ${ }^{[27]}$ reported that $32 \%$ of PD patients in the surgery group had developed dementia at a final follow-up of 2 years, compared with $16 \%$ in the non-surgery group. These findings to some extent indicate that the changes in intelligence and memory are likely to be consequences of the natural evolution of PD.

The data in Table 1 also indicate that patients with a high education level suffered memory declines, whereas those with a low education level did not, possibly because the former had larger vocabulary reserves at baseline ${ }^{[14]}$.

\section{Attention}

Trail-making test $A$ was used to evaluate PD patients undergoing STN-DBS, and revealed reduced attention compared with pre-operation ${ }^{[8]}$ and a control group ${ }^{[26]}$, consistent with several previous studies ${ }^{[20,21]}$. Zibetti et al. ${ }^{[18]}$ used trail-making test $B$ to evaluate attentional shifting, reporting that it was elevated at one year post-surgically, returned to baseline at 5 years, then was significantly reduced within 9 years. However, a multicenter control study revealed no significant difference in attentional shifting between surgery and non-surgery groups, as evaluated using the Mattis dementia rating scale ${ }^{[29]}$. As shown above, the attention profile after STN-DBS is also a controversial issue. Apart from different scale sensitivities and follow-up durations, differences in baseline data, such as age, duration and severity of disease, and drug dose, may also contribute to the differences in findings.

\section{Other Cognitive Domains}

Psychomotor speed and spatial visual function were reported to show no significant pre- versus post-operative changes following STN-DBS ${ }^{[13,20-27]}$. However, one study reported that STN-DBS reduced psychomotor speed in PD patients and improved spatial visual function, while another reported increased psychomotor speed after surgery ${ }^{[30]}$. In addition, information-processing velocity ${ }^{[27]}$, computation ${ }^{[8]}$, and procedural learning ${ }^{[31]}$ were also reported to be reduced following STN-DBS.

In general, cognition embraces comprehensive domains. To date, there is no international consensus on screening instruments, so some findings might not be generalizable to all PD patients with STN-DBS. So in future, more attention must be paid to the need for uniform neuropsychological scales and screening instruments, baseline patient data, and follow-up durations.

\section{Related Mechanisms}

The precise nature of the changes in cognitive function exhibited by PD patients after DBS is currently unclear. Clinical studies ${ }^{[32,33]}$ and positron emission tomography experiments $^{[34]}$ have reported that the basal ganglia participate in cognition and emotion as well as self-initiated 
movement, functioning as a network that connects the parallel loops between the thalamus and cortex. The structures and functions of these parallel loops appear to be distinct, with each loop originating from a specific region of the prefrontal lobe and connecting with different sites in the basal ganglia, then returning to the frontal lobe via the thalamus. The basal ganglia-thalamus-dorsal prefrontal lobe loop is thought to constitute the anatomical and physiological substrate of cognitive function ${ }^{[35]}$. STN projection fibers are the only excitatory connections in the basal ganglia and play an important role in regulating $\operatorname{cognition}^{[32,36,37]}$. Changes in each part of the loop can alter cognitive function. Several explanations for the cognitive changes following DBS have been proposed.

\section{Surgery-induced Micro-injury}

A clinical study reported that electrode positioning and the surgical approach can induce cognitive changes, some of which only occur in the early postoperative stage $^{[23]}$. Lefaucheur et al. ${ }^{[38]}$ evaluated verbal fluency preoperatively, and at 3,10 , and 180 days after operation, reporting that micro-injury during surgery reduces verbal fluency. However, a logistic analysis of follow-up data revealed that micro-injury was not a predictive factor in cognitive impairment ${ }^{[26]}$. All of the studies above suggest that any surgical DBS approach involving the basal ganglia-thalamus-dorsal prefrontal lobe loop may impair cognitive function. To elucidate this question, future studies should focus on animals subjected to sham surgery or stimulation.

\section{Current Stimulation}

Since the STN is small and its projection fibers are relatively concentrated, a stimulus current may travel from the motor area of the STN to non-motor areas, influencing the basal ganglia-thalamus-dorsal prefrontal lobe loop. This proposal is supported by the finding that highfrequency stimulation leads to significantly reduced verbal fluency compared with low-frequency stimulation ${ }^{[39]}$.

\section{Reduced Dose of Postoperative Dopaminergic Drugs}

There are direct and indirect pathways between the cortex and basal ganglia and dopamine regulates both, suggesting that it influences cognition ${ }^{[40,41]}$, including verbal fluency, working memory, shifting, problem-solving, and planning ${ }^{[41]}$. Saint-Cyr et al. ${ }^{[42]}$ reported that postoperative decrements of dopaminergic drugs resulted in psychological symptoms like apathy and depression, possibly affecting the results of other neuropsychological tests and influencing cognitive function. This notion is supported by another report ${ }^{[14]}$ showing that a greater age at onset, duration of education, and dose of dopaminergic drugs are associated with a reduced likelihood of cognitive decline. In contrast, several studies have reported no association between cognitive changes and a reduction in dopaminergic drug intake ${ }^{[20,26,29]}$. Thus, further studies are needed.

\section{PD Progression}

Moreover, the natural course of PD itself cannot be ignored; this may also lead to cognitive dysfunction, since a majority of patients at moderate or advanced stage of PD frequently suffer from non-motor symptoms such as a progressive decline of cognitive functions leading to dementia ${ }^{[43]}$. A recent long-term follow-up study reported that the incidence of dementia in PD patients over 3 years after STN-DBS is similar to that in those treated medically ${ }^{[8]}$. To some extent, this indicates that some cognitive profiles are likely compatible with the natural evolution of PD. However, these cannot completely deny the effect of STN-DBS on cognition, because more and more controlled studies and even multi-center, randomized control experiments tend to reach a consensus that there is a significant change in the DBS-treated group compared with the control group, although both groups show progressive worsening in some studies. On the basis of our findings on one point of verbal fluency (unpublished data), we are inclined to support the position that surgical intervention, rather than natural progression, underlies the changes. However, with regard to long-term executive function and memory, the natural course of progression of PD is more likely to contribute to the differences. More controlled, randomized studies with long follow-up periods are needed to test our hypothesis.

In summary, a variety of loops and factors are involved in the DBS-induced changes of cognitive function, but further studies are required.

\section{Conclusions and Prospects}

A growing number of studies have investigated the influence of DBS on cognitive function. These studies vary in terms of the regions examined and the durations of follow-up: short-term (6 months to 1 year), medium-term 
(2-5 years), and long-term (more than 5 years, up to 9 years) (Table 1). Moreover, the cognitive function category and strength of evidence used differ among studies. Most previous studies were self-controlled cohort studies, without a non-surgery control group. Some cohort studies involved a non-surgery control group, but did not use randomization. Only one multi-center, randomized, controlled clinical experiment has been conducted ${ }^{[29]}$, but the follow-up duration was only 6 months. Previous studies ${ }^{[44]}$ involving follow-up of PD patients subjected to STN-DBS in China have mainly examined motor function; only one such study reported that language function was not significantly improved at 3 years after STN-DBS ${ }^{[45]}$.

In conclusion, verbal fluency is consistently reported to progressively decrease after STN-DBS; executive function is unchanged in the intermediate postoperative stage (1-2 years), while in the early ( $<6$ months) and later stages ( $>5$ years) it tends to decline. Changes in other domains such as intelligence and memory, attention, psychomotor speed, and spatial visual function remain controversial. However, there are some limitations in these clinical studies which should not be ignored. Sample sizes and test validity were often relatively low, so the results may be not representative. In addition, many studies did not use control groups or randomization, reducing the strength of the evidence. Double-blind designs have not been commonly used, so subjective factors may have influenced the results. Moreover, sample sizes and baseline data in some studies were not matched. Follow-up periods were often short, few studies extending longer than 5 years. Thus, further research involving regional cooperation to conduct multicenter, randomized, controlled, long-term follow-up studies should be conducted to obtain valuable results.

Although verbal fluency is reported to be reduced following STN-DBS, this reduction does not appear to decrease the overall quality of life, because STN-DBS improves the motor symptoms, which may offset the negative emotional impact of cognitive decline. So STNDBS seems to be safe with respect to cognitive effects in carefully-selected patients during a certain follow-up period from 6 months to 9 years. However, cognitive changes should not be disregarded. Thus, a computer-based model that can visualize electrode position and regulate stimulus parameters would be a valuable contribution ${ }^{[46]}$ to maximize the control of motor symptoms, and minimize non-motor symptoms using minimal current levels.

\section{ACKNOWLEDGMENTS}

We thank Professor Hallett for revision of this manuscript. This review was supported by National Natural Science Foundation of China (81071065).

Received date: 2012-12-23; Accepted date: 2013-04-05

\section{REFERENCES}

[1] Kleiner-Fisman G, Herzog J, Fisman DN, Tamma F, Lyons $\mathrm{KE}$, Pahwa R, et al. Subthalamic nucleus deep brain stimulation: summary and meta-analysis of outcomes. Mov Disord 2006, 21 (Suppl 14): S290-304.

[2] Sun B, Chen S, Zhan S, Le W, Krahl SE. Subthalamic nucleus stimulation for primary dystonia and tardive dystonia. Acta Neurochir Suppl 2007, 97: 207-214.

[3] Phillips L, Litcofsky KA, Pelster M, Gelfand M, Ullman MT, Charles PD. Subthalamic nucleus deep brain stimulation impacts language in early Parkinson's disease. PLoS One 2012, 7: e42829.

[4] Parsons TD, Rogers SA, Braaten AJ, Woods SP, Troster Al. Cognitive sequelae of subthalamic nucleus deep brain stimulation in Parkinson's disease: a meta-analysis. Lancet Neurol 2006, 5: 578-588.

[5] Castelli L, Perozzo P, Zibetti M, Crivelli B, Morabito U, Lanotte $\mathrm{M}$, et al. Chronic deep brain stimulation of the subthalamic nucleus for Parkinson's disease: effects on cognition, mood, anxiety and personality traits. Eur Neurol 2006, 55: 136-144.

[6] De Gaspari D, Siri C, Di Gioia M, Antonini A, Isella V, Pizzolato A, et al. Clinical correlates and cognitive underpinnings of verbal fluency impairment after chronic subthalamic stimulation in Parkinson's disease. Parkinsonism Relat Disord 2006, 12: 289-295.

[7] Erola T, Heikkinen ER, Haapaniemi T, Tuominen J, Juolasmaa A, Myllyla VV. Efficacy of bilateral subthalamic nucleus (STN) stimulation in Parkinson's disease. Acta Neurochir (Wien) 2006, 148: 389-394.

[8] Aybek S, Gronchi-Perrin A, Berney A, Chiuve SC, Villemure JG, Burkhard PR, et al. Long-term cognitive profile and incidence of dementia after STN-DBS in Parkinson's disease. Mov Disord 2007, 22: 974-981.

[9] Castelli L, Lanotte M, Zibetti M, Caglio M, Rizzi L, Ducati A, et al. Apathy and verbal fluency in STN-stimulated PD patients. An observational follow-up study. J Neurol 2007 , 254: 1238-1243. 
[10] Ory-Magne F, Brefel-Courbon C, Simonetta-Moreau M, Fabre $\mathrm{N}$, Lotterie JA, Chaynes $\mathrm{P}$, et al. Does ageing influence deep brain stimulation outcomes in Parkinson's disease? Mov Disord 2007, 22: 1457-1463.

[11] Rothlind JC, Cockshott RW, Starr PA, Marks WJ Jr. Neuropsychological performance following staged bilateral pallidal or subthalamic nucleus deep brain stimulation for Parkinson's disease. J Int Neuropsychol Soc 2007, 13: 68-79.

[12] Contarino MF, Daniele A, Sibilia AH, Romito LM, Bentivoglio AR, Gainotti G, et al. Cognitive outcome 5 years after bilateral chronic stimulation of subthalamic nucleus in patients with Parkinson's disease. J Neurol Neurosurg Psychiatry 2007, 78: 248-252.

[13] Klempirova O, Jech R, Urgosik D, Klempir J, Spackova $\mathrm{N}$, Roth J, et al. Deep brain stimulation of the subthalamic nucleus and cognitive functions in Parkinson's disease. Prague Med Rep 2007, 108: 315-323.

[14] Heo JH, Lee KM, Paek SH, Kim MJ, Lee JY, Kim JY, et al. The effects of bilateral subthalamic nucleus deep brain stimulation (STN DBS) on cognition in Parkinson disease. J Neurol Sci 2008, 273: 19-24.

[15] Fasano A, Romito LM, Daniele A, Piano C, Zinno M, Bentivoglio AR, et al. Motor and cognitive outcome in patients with Parkinson's disease 8 years after subthalamic implants. Brain 2010, 133: 2664-2676.

[16] Kishore A, Rao R, Krishnan S, Panikar D, Sarma G, Sivasanakaran MP, et al. Long-term stability of effects of subthalamic stimulation in Parkinson's disease: Indian Experience. Mov Disord 2010, 25: 2438-2444.

[17] Merola A, Zibetti M, Angrisano S, Rizzi L, Ricchi V, Artusi CA, et al. Parkinson's disease progression at 30 years: a study of subthalamic deep brain-stimulated patients. Brain 2011, 134: 2074-2084.

[18] Zibetti M, Merola A, Rizzi L, Ricchi V, Angrisano S, Azzaro C, et al. Beyond nine years of continuous subthalamic nucleus deep brain stimulation in Parkinson's disease. Mov Disord 2011, 26: 2327-2334.

[19] Yamanaka T, Ishii F, Umemura A, Miyata M, Horiba M, Oka $Y$, et al. Temporary deterioration of executive function after subthalamic deep brain stimulation in Parkinson's disease. Clin Neurol Neurosurg 2012, 114: 347-351.

[20] Smeding HM, Speelman JD, Koning-Haanstra M, Schuurman PR, Nijssen P, van Laar T, et al. Neuropsychological effects of bilateral STN stimulation in Parkinson disease: a controlled study. Neurology 2006, 66: 1830-1836.

[21] York MK, Dulay M, Macias A, Levin HS, Grossman R, Simpson $\mathrm{R}$, et al. Cognitive declines following bilateral subthalamic nucleus deep brain stimulation for the treatment of Parkinson's disease. J Neurol Neurosurg Psychiatry 2008, 79: 789-795.
[22] Zahodne LB, Okun MS, Foote KD, Fernandez HH, Rodriguez $\mathrm{RL}$, Kirsch-Darrow $\mathrm{L}$, et al. Cognitive declines one year after unilateral deep brain stimulation surgery in Parkinson's disease: a controlled study using reliable change. Clin Neuropsychol 2009, 23: 385-405.

[23] Zangaglia R, Pacchetti C, Pasotti C, Mancini F, Servello $D$, Sinforiani E, et al. Deep brain stimulation and cognitive functions in Parkinson's disease: A three-year controlled study. Mov Disord 2009, 24: 1621-1628.

[24] Castelli L, Rizzi L, Zibetti M, Angrisano S, Lanotte M, Lopiano L. Neuropsychological changes 1-year after subthalamic DBS in PD patients: A prospective controlled study. Parkinsonism Relat Disord 2010, 16: 115-118.

[25] Alegre M, Hallett M, Olanow CW, Obeso JA. Technical advances in deep brain stimulation: how far is enough? Mov Disord 2012, 27: 341-342.

[26] Smeding HM, Speelman JD, Huizenga HM, Schuurman PR, Schmand B. Predictors of cognitive and psychosocial outcome after STN DBS in Parkinson's Disease. J Neurol Neurosurg Psychiatry 2011, 82: 754-760.

[27] Williams AE, Arzola GM, Strutt AM, Simpson R, Jankovic $\mathrm{J}$, York MK. Cognitive outcome and reliable change indices two years following bilateral subthalamic nucleus deep brain stimulation. Parkinsonism Relat Disord 2011, 17: 321-327.

[28] Zangaglia R, Pasotti C, Mancini F, Servello D, Sinforiani E, Pacchetti C. Deep brain stimulation and cognition in Parkinson's disease: an eight-year follow-up study. Mov Disord 2012, 27: 1192-1194.

[29] Witt K, Daniels C, Reiff J, Krack P, Volkmann J, Pinsker MO, et al. Neuropsychological and psychiatric changes after deep brain stimulation for Parkinson's disease: a randomised, multicentre study. Lancet Neurol 2008, 7: 605-614.

[30] Funkiewiez A, Ardouin C, Caputo E, Krack P, Fraix V, Klinger $\mathrm{H}$, et al. Long term effects of bilateral subthalamic nucleus stimulation on cognitive function, mood, and behaviour in Parkinson's disease. J Neurol Neurosurg Psychiatry 2004, 75: 834-839.

[31] Wang XP, Sun BM, Ding HL. Changes of procedural learning in Chinese patients with non-demented Parkinson disease. Neurosci Lett 2009, 449: 161-163.

[32] Balaz M, Rektor I, Pulkrabek J. Participation of the subthalamic nucleus in executive functions: An intracerebral recording study. Mov Disord 2008, 23: 553-557.

[33] Jiang Y, Shen X, Wang X, Li W. Procedural learning changes in patients with Wilson's disease. Neur Regen Res 2011, 6(18): 1410-1414.

[34] Kalbe E, Voges J, Weber T, Haarer M, Baudrexel S, Klein $\mathrm{JC}$, et al. Frontal FDG-PET activity correlates with cognitive outcome after STN-DBS in Parkinson disease. Neurology 2009, 72: 42-49. 
[35] Volkmann J, Daniels C, Witt K. Neuropsychiatric effects of subthalamic neurostimulation in Parkinson disease. Nat Rev Neurol 2010, 6: 487-498.

[36] Naskar S, Sood SK, Goyal V, Dhara M. Mechanism(s) of deep brain stimulation and insights into cognitive outcomes in Parkinson's disease. Brain Res Rev 2010, 65: 1-13.

[37] Wang XP, Ding HL. Alzheimer's disease: epidemiology, genetics, and beyond. Neurosci Bull 2008, 24: 105-109.

[38] Lefaucheur R, Derrey S, Martinaud O, Wallon D, Chastan $\mathrm{N}$, Gerardin E, et al. Early verbal fluency decline after STN implantation: is it a cognitive microlesion effect? J Neurol Sci 2012, 321: 96-99.

[39] Wojtecki L, Timmermann L, Jorgens S, Sudmeyer M, Maarouf $\mathrm{M}$, Treuer $\mathrm{H}$, et al. Frequency-dependent reciprocal modulation of verbal fluency and motor functions in subthalamic deep brain stimulation. Arch Neurol 2006, 63: 1273-1276.

[40] Hanna-Pladdy B, Heilman KM. Dopaminergic modulation of the planning phase of skill acquisition in Parkinson's disease. Neurocase 2010, 16: 182-190.

[41] Romito LM, Albanese A. Dopaminergic therapy and subthalamic stimulation in Parkinson's disease: a review of 5-year reports. J Neurol 2010, 257: S298-304.

[42] Saint-Cyr JA, Trepanier LL. Neuropsychologic assessment of patients for movement disorder surgery. Mov Disord 2000, 15: 771-783.

[43] Xia R, Mao ZH. Progression of motor symptoms in Parkinson's disease. Neurosci Bull 2012, 28: 39-48.

[44] Liang J, Hu X, Zhou X, Jiang X, Cao Y, Wang L, et al. Fiveyear follow-up of 23 asymmetrical Parkinson's disease patients treated with unilateral subthalamic nucleus stimulation. Neural Regen Res 2012, 7(1): 1428-1435.

[45] Zhang KZ, Wan Q, Zhang L, Yuan YS. Valuation of therapeutic effects and safety of bilateral stimulation of the subthalamic nucleus in improving dyskinesia on "Off" time of Parkinson's disease. Chin J Clin Neurosci 2012, 20(3): 290-294. [Article in Chinese]

[46] Frankemolle AM, Wu J, Noecker AM, Voelcker-Rehage C, Ho $\mathrm{JC}$, Vitek JL, et al. Reversing cognitive-motor impairments in Parkinson's disease patients using a computational modelling approach to deep brain stimulation programming. Brain 2010, 133: 746-761. 